\title{
Slow dynamics of thin nematic films in the presence of adsorbed nanoparticles
}

\author{
S. Grollau, O. Guzmán, N. L. Abbott, and J. J. de Pabloa) \\ Department of Chemical and Biological Engineering, University of Wisconsin, Madison, \\ Wisconsin 53706-1619
}

(Received 14 January 2004; accepted 21 October 2004; published online 17 December 2004)

\begin{abstract}
Recent experiments indicate that liquid crystals can be used to optically report the presence of biomolecules adsorbed at solid surfaces. In this work, numerical simulations are used to investigate the effects of biological molecules, modeled as spherical particles, on the structure and dynamics of nematic ordering. In the absence of adsorbed particles, a nematic in contact with a substrate adopts a uniform orientational order, imposed by the boundary conditions at this surface. It is found that the relaxation to this uniform state is slowed down by the presence of a small number of adsorbed particles. However, beyond a critical concentration of adsorbed particles, the liquid crystal ceases to exhibit uniform orientational order at long times. At this concentration, the domain growth is characterized by a first regime where the average nematic domain size $L_{D}$ obeys the scaling law $L_{D}(t) \sim t^{1 / 2}$; at long times, a slow dynamics regime is attained for which $L_{D}$ tends to a finite value corresponding to a metastable state with a disordered texture. The results of simulations are consistent with experimental observations. (C) 2005 American Institute of Physics.
\end{abstract}

[DOI: $10.1063 / 1.1831253]$

\section{INTRODUCTION}

Recent experiments have shown that liquid crystals (LC) can be used to report ligand-receptor binding at solid surfaces. ${ }^{1-4}$ Proteins or viruses bound to solid surfaces can create elastic deformations of a thin nematic liquid-crystal film giving rise to macroscopic optical signatures that can be easily detected with crossed polarizers. ${ }^{1-4}$ These experiments constitute the basis for liquid-crystal based biosensors.

The occurrence of an optical signature relies on a nonequilibrium process where the binding of biomolecules to a solid surface influences the relaxation of the liquid crystal. Understanding the effects of adsorbed particles on the structure and dynamics of a nematic LC would facilitate considerably the development of such sensors. One of the questions raised by these experiments is how can events that occur on a nanometer scale influence the mesoscopic optical response of the liquid crystal.

In a series of papers, ${ }^{5-7}$ a multiscale approach was used to investigate systems of a few nanoscopic colloids immersed in a nematic host. This approach combines molecular simulation and a dynamic field theory (DyFT) for the tensor order parameter. The coarse-grained description offered by the DyFT was seen to describe correctly the mean force between colloids, and the topological defect structures around them (provided that the size and separation of the nanoscopic particles are at least on the order of a few molecular sizes). ${ }^{6,7}$ A fully molecular simulation of a biosensor is beyond our current computational power. Therefore, in this work we turn to a dynamic field theory description of a model biosensor in order to address the effects of nanometer size, adsorbed par-

a)Electronic mail: depablo@engr.wisc.edu ticles on the structure, and ordering dynamics of nematic films.

When a system is quenched from a disordered equilibrium state into an ordered phase, the ensuing nonequilibrium state slowly evolves in the form of domains of ordered phase that grow as a function of time. Coarsening dynamics in nematic LCs have been the subject of numerous experimental observations ${ }^{8,9}$ and numerical simulations; ${ }^{10-15}$ considerable controversy has emerged as to whether the ordering violates dynamical scaling and whether at late stages the system enters a regime characterized by a single length scale $L(t)$. As pointed out in previous studies based on cell-dynamics simulations, ${ }^{10,11}$ numerical determination of growth exponents may be affected by transient effects or by the freezing of the system into a metastable configuration caused by numerical pinning of topological defects.

This work studies the ordering dynamics of a twodimensional macroscopic nematic film, confined between two substrates in the presence of nanoscopic particles adsorbed at these substrates (see Fig. 1). In the absence of particles, at late stages, the confining surfaces promote a uniform nematic order; we investigate the effects of the adsorbed particles on the equilibrium structure and dynamics. The characteristic dimensions of the film are large compared to the nematic coherence length $(\xi \approx 20 \mathrm{~nm}),{ }^{16,17}$ while the size of the nanoparticles is on the order of a few coherence lengths: the biological particles that are the targets of the experimental sensors have a size on the order of tens or hundreds of nanometers, hence in the model sensor that we present the adsorbed particles have a radius $R=2.4 \xi$, which is large compared to the coherence length of the bulk nematic. The relaxation of the nematic is described by a time evolution equation for the tensor order parameter ${ }^{17} \mathrm{Q}$ (also called alignment tensor) that includes both short-range and 


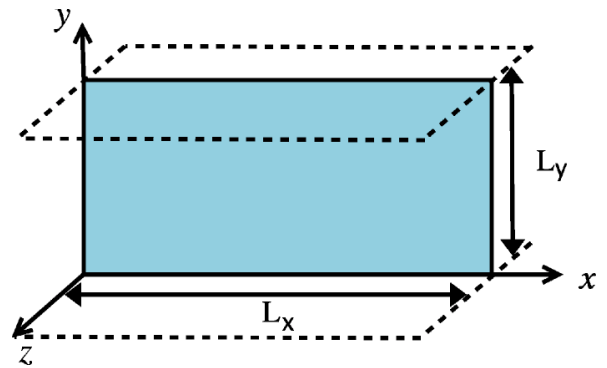

FIG. 1. A schematic view of the model sensor: a film of nematic is confined by two plane surfaces (dashed lines). A quasi-2D geometry is obtained by taking the fluid as invariant along the $z$ direction. We compute the evolution of the LC on a rectangle of size $L_{x} \times L_{y}$, periodic boundary conditions are assumed in the $x$ direction.

long-range order elastic effects. ${ }^{18}$ The tensor order parameter formulation has been successfully applied to study the defect structure around an individual particle, ${ }^{19,20}$ or a group of particles. $6,7,21$

The system is first equilibrated in the isotropic phase; after a sudden quench into the nematic phase, its evolution is analyzed through the correlation function of the tensor order parameter for different concentrations of particles. Simulations are performed with sufficiently high accuracy to avoid artificial numerical pinning of the topological defects.

\section{DESCRIPTION OF THE MODEL}

We compute the evolution of the liquid crystal with a dynamic field theory for the tensor order parameter $\mathrm{Q}(\mathbf{r}, t)$. This theory corresponds to a particular case of the BerisEdwards formulation of the thermodynamics of fluids with internal microstructure. ${ }^{18}$ In this theory, the functional derivative of the free energy $\mathcal{F}$ with respect to the order parameter drives the evolution of $\mathrm{Q}$ :

$$
\frac{\partial \mathrm{Q}}{\partial t}=-\Gamma\left[\frac{\delta \mathcal{F}}{\delta \mathrm{Q}}-\frac{1}{3} \operatorname{Tr}\left(\frac{\delta \mathcal{F}}{\delta \mathrm{Q}}\right) \mathrm{I}\right] .
$$

The coefficient $\Gamma=6 D /\left[1-3 \operatorname{Tr}\left(\mathrm{Q}^{2}\right) / 2\right]^{2}$, where $D$ is the rotational diffusion for the nematic, and $\mathrm{I}$ is the identity tensor. Note that $\delta \mathcal{F} / \delta \mathrm{Q}$ must be symmetrized.

The free energy $\mathcal{F}$ of the liquid crystal includes two contributions. One corresponds to the short-range bulk interactions responsible for the I-N transition; the other one arises from the elasticity of the liquid crystal,

$$
\mathcal{F}=\int_{V}\left(F_{s}+F_{e}\right) \mathrm{d} \mathbf{r} .
$$

In the experiments with the LC sensor, ${ }^{1-4}$ the orientation of the liquid crystal at the substrates (referred to as planar or homeotropic anchoring) as well as the strength of anchoring are controlled through specific treatments based on chemisorption of alkanethiols on gold surfaces. ${ }^{22}$

This work only considers the case of strong anchoring at all surfaces; surface free energy contributions need not be included, provided that the initial configurations satisfy the anchoring at every confining surface.
The short-range free energy density is represented with a Landau-de Gennes expansion. In particular, we use the Doi theory expression ${ }^{23}$

$$
F_{s}=\int \frac{A}{2}\left(1-\frac{U}{3}\right) \operatorname{tr}\left(\mathrm{Q}^{2}\right)-\frac{A U}{3} \operatorname{tr}\left(\mathrm{Q}^{3}\right)+\frac{A U}{4} \operatorname{tr}\left(\mathrm{Q}^{2}\right)^{2} \mathrm{~d} \mathbf{r},
$$

because this will allow future comparisons with previous work on phase ordering that also use this model. ${ }^{15,24}$ It contains only two phenomenological coefficients $(A$ and $U)$ that depend on the liquid crystal of interest; $A$ controls the energy scale of the model, while $U$ controls the value of the scalar order parameter in the bulk, ${ }^{18}$

$$
S_{\text {bulk }}(U)=\frac{1}{4}+\frac{3}{4} \sqrt{1-\frac{8}{3 U}} .
$$

In this model, the system is isotropic for $0<U<U_{I N}=2.7$, and nematic for $U>U_{I N}$. Since the coefficient of the quartic term is not independent from $A$ and $U$, the jump in the scalar order parameter at the transition is fixed. The limits of metastability for the isotropic and nematic phases are $U^{*}$ $=3$ and $U^{* *}=8 / 3$, respectively.

The elastic contribution introduces a free energy cost for gradients in the tensor order parameter field. Using the notation $Q_{i j, k}=\partial_{k} Q_{i j}$, this contribution is given by ${ }^{25}$

$$
\begin{aligned}
F_{e}= & \int \frac{L_{1}}{2} Q_{i j, k} Q_{i j, k}+\frac{L_{2}}{2} Q_{i k, i} Q_{j k, j} \\
& +\frac{L_{3}}{2} Q_{i j} Q_{k l, i} Q_{k l, j} \mathrm{~d} \mathbf{r} .
\end{aligned}
$$

The coefficients $L_{i}$ are related to the splay $\left(K_{1}\right)$, twist $\left(K_{2}\right)$, and bend $\left(K_{3}\right)$ elastic constants through

$$
\begin{aligned}
& L_{1}=\frac{3 K_{2}-K_{1}+K 3}{6 S^{2}}, \\
& L_{2}=\frac{K_{1}-K_{2}}{S^{2}}, \\
& L_{3}=\frac{K_{3}-K_{1}}{2 S^{3}} .
\end{aligned}
$$

In this work, we use the one-elastic-constant approximation, ${ }^{17,26}$ where the splay, twist, and bend elastic constants have a common value $K$. In this approximation, the elastic free energy density reduces to

$$
F_{e}=\int \frac{L_{1}}{2} Q_{i j, k} Q_{i j, k} \mathrm{~d} \mathbf{r},
$$

while the elastic constant depends quadratically on the scalar order parameter, ${ }^{27}$

$$
K=2 S^{2} L_{1} \text {. }
$$

When the functional derivatives in Eq. (1) are evaluated, one obtains a partial differential equation for $Q$,

$$
\begin{aligned}
\frac{\partial \mathrm{Q}}{\partial t}= & -\Gamma\left\{A(1-U / 3) \mathrm{Q}-A U\left[\mathrm{Q}^{2}-\operatorname{Tr}\left(\mathrm{Q}^{2}\right)(\mathrm{Q}+\mathrm{l} / 3)\right]\right. \\
& \left.-L_{1} \nabla^{2} \mathrm{Q}\right\} .
\end{aligned}
$$


We introduce the nematic coherence length $\xi$ defined by $\xi=\sqrt{18 L_{1} / A U} ; \xi$ provides a characteristic length scale for changes of the order parameter. At the isotropic-nematic first-order transition, $\xi$ is finite and amounts to several molecular lengths, with $\xi \sim 20 \mathrm{~nm} .{ }^{16}$ In the nematic phase, $\xi$ also amounts to several molecular lengths and corresponds to the characteristic spatial extension of the core defects. ${ }^{28,20}$ We also introduce $\tau_{0}=|6 D A(1-U / 3)|^{-1}$, corresponding to the characteristic time scale for changes of the order parameter. For the parameter values used in this work, ${ }^{29}$ the relaxation time $\tau$ is on the order of $100 \mathrm{~ns}$. In the following, lengths are given in units of $\xi$ evaluated in the nematic phase at $U=6$ and time is given in units of $\tau_{0}$.

The numerical study presented in this work is restricted to the case of strong, planar anchoring along the $x$ direction at the top and bottom solid surfaces (see Fig. 1). At all bounding surfaces, we assume $Q$ to be uniaxial and of the form

$$
\mathrm{Q}=S_{\text {bulk }}(\mathbf{n n}-\mathrm{l} / 3),
$$

where $\mathbf{n}$ is the average orientation of the molecules at the surfaces. Equation (12) indicates that the substrate is treated as a rigid surface with well defined anchoring properties. This is a simplification over the more realistic situation where the surface anchoring is explicitly modeled over distances that are small compared to the surface correlation length ${ }^{30}$ but since the focus of this work is to analyze the behavior of the liquid crystal in the central regions of the sensor, we believe this simplification is adequate.

Past studies have documented how an assembly of phospholipids at the interface of a liquid crystal can induce planar or homeotropic anchoring depending on the organization and tilt of the phospholipids comprising the assembly. ${ }^{31,32}$ More recently, it has been found that enveloped viruses (such as the vesicular stomatitis virus) can induce homeotropic anchoring of liquid crystals when deposited on gold films treated with poly-L-lysine (cationic surfaces) that normally induce planar anchoring. ${ }^{33,34}$ (We note that, even if the composition of the virion's envelope is relatively well characterized, the detailed mechanism by which homeotropic anchoring is obtained is not well understood.) Since viral particles are important targets for a biosensor, in this work we consider the case where the liquid crystal director is oriented perpendicularly to the surface of the nanoparticles (strong homeotropic anchoring).

The geometry of the simulation cell corresponds to a two-dimensional thin film, with lengths $L_{x}=290 \xi$ and a ratio $L_{y} / L_{x}=1 / 6$ (see Fig. 1). Periodic boundary conditions are imposed in the $x$ direction. In order to avoid artificial numerical pinning of the defects, we use a grid of $1024 \times 171$ points. This mesh allows us to observe a uniform orientation texture, free of topological defects, when the liquid crystal relaxes between the two surfaces in the absence of particles.

The biological molecules adsorbed at the substrates are modeled by spherical particles fixed at the confining walls. The radius of the particles is $R=2.4 \xi$; this radius is representative of large proteins or small viruses. In the following, we use $\phi=2 R N / L_{x}$ to denote the dimensionless concentration of $N$ particles adsorbed on each surface. To solve Eq.
(11) for specific boundary conditions in the presence of particles, we use a numerical algorithm described in recent work. ${ }^{5,20}$ The initial configuration corresponds to a random orientation of the director. The system is equilibrated in the isotropic phase at the nematic potential $U=2$ for a time $t$ $=20 \tau_{0}$. The system is quenched deep into the nematic phase at the nematic potential $U=6$, and its time evolution is quantified along the $x$ direction and in the middle of the cell through the scalar correlation function defined by

$$
C(x, t)=\frac{\langle\operatorname{Tr}[\mathrm{Q}(x, t) \mathrm{Q}(0, t)]\rangle}{\langle\operatorname{Tr}[\mathrm{Q}(0, t) \mathrm{Q}(0, t)]\rangle},
$$

where $\langle\cdots\rangle$ denotes an average over positions. The correlation function is normalized so that $C(0, t)=1$; we compute the average domain size ${ }^{11} L_{D}(t)$ at time $t$ as the solution to the equation

$$
C\left(L_{D}, t\right)=1 / 2 .
$$

\section{EFFECTS OF THE ADSORBED PARTICLES}

Figure 2 shows the evolution of the correlation function $C(x, t)$ for different values of the concentration of adsorbed particles. For each concentration, $C(x, t)$ is plotted at a sequence of time steps $\left(t / \tau_{0}=0,1.4,4.2,5.6,7,17.5,35,70\right.$, $105,175,280,350,525$, and 630). In all the cases, a first regime is observed from $t=0$ to $t \simeq 105 \tau_{0}$. In this regime, the correlation function decays to zero within a few $\xi$. The size of the domains in the middle of the cell is small and grows as a result of the liquid crystal's propensity to align in a given orientation, but this direction is not yet dictated by the confining surfaces.

Only at later times $\left(t>105 \tau_{0}\right)$ does the preferred orientation imparted by the surfaces get transmitted to the middle of the cell. In this stage, the correlation function no longer decays to zero at long distances, but tends to a "background" value, independent of position, that increases monotonically over time. In the absence of adsorbed particles [Fig. 2(a)] by the time $t=650 \tau_{0}$ the correlation function approaches unity over the entire cell, indicating the existence of a uniform texture driven by the substrates' anchoring properties.

However, the presence of particles slows down the dynamics at late stages, as shown in Figs. 2(b)-2(d). The rate at which the background value approaches unity decreases with increasing $\phi$. In particular, for $\phi \geqslant 0.33$ [see Figs. 2(c) and 2(d)], the background value of the correlation function at large separations never becomes higher than $1 / 2$, even at late stages; and the domain size $L_{D}$ [defined in Eq. (14)] remains finite.

Two snapshots of the simulation cell with particles at the substrates are shown in Fig. 3 for the concentration $\phi$ $=0.33$. The system is visualized as in between crossed polars: the intensity of light is proportional to $\sin ^{2}(2 \theta)$, where $\theta$ is the angle between the local director and the $x$ axis; thus, regions with vertical or horizontal directors appear as black. For this particle configuration, the structure of the nematic at late stages is characterized by the presence of isolated topological defects that move slowly through the cell, and topological defects attached to the particles. More importantly, as indicated by the occurrence of alternating white and black 

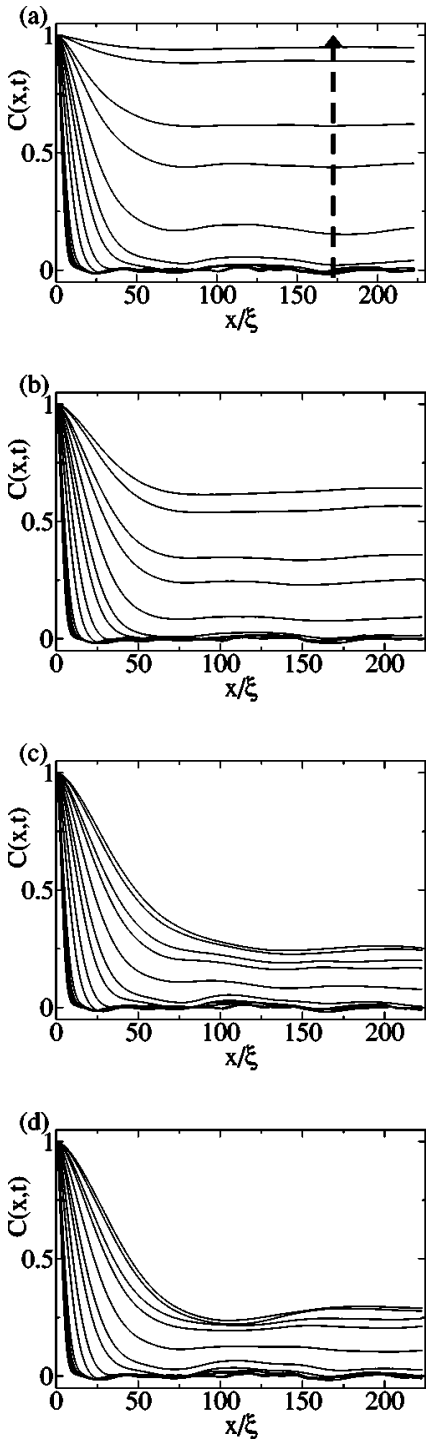

FIG. 2. The correlation function $C(x, t)$ at a sequence of time steps $\left(t / \tau_{0}\right.$ $=0,1.4,4.2,5.6,7,17.5,35,70,105,175,280,350,525$, and 630) for four concentrations of particles: (a) $\phi=0$, (b) $\phi=0.17$, (c) $\phi=0.33$, and (d) $\phi$ $=0.41$. The arrow indicates the order of increasing time.

"bridges" between the two substrates (see the optical picture at $t=315 \tau_{0}$ ), the presence of bound particles induces considerable disorder in the liquid crystal after relaxation. It is interesting to compare these banded structures with the textures observed by Rey and Tsuji in their simulations of liquid crystal films after cessation of flow. ${ }^{24}$ In those simulations, a simple rectilinear shear flow gives rise to banded textures,

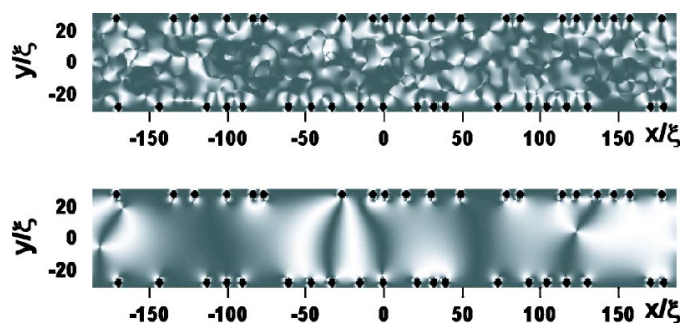

FIG. 3. Nematic ordering in the presence of particles adsorbed at the substrates: optical pictures at $t=5.6 \tau_{0}$ (top) and $t=315 \tau_{0}$ (bottom).

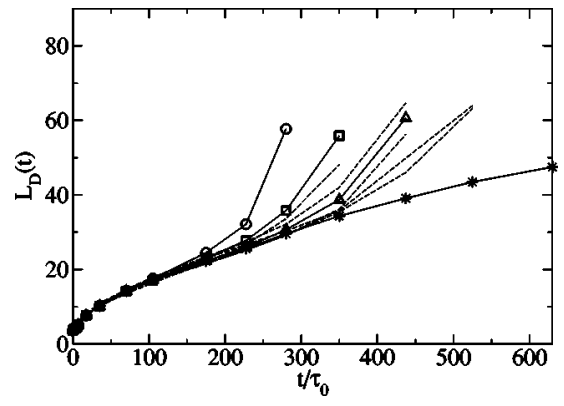

FIG. 4. Growth of domain size $L_{D}(t)$ without particles (circles), with $\phi$ $=0.08$ (squares), with $\phi=0.17$ (triangles), and with $\phi=0.33$ (star). Statistical averages are performed over five different realizations of the particles' positions. For each realization, averages are performed over 30 initial configurations of the liquid crystal. The dashed lines represent $L_{D}(t)$ for the five different realizations with $\phi=0.17$.

which are ultimately associated with an out-of-plane director orientation; after cessation of the flow, the banded texture relaxes towards a uniform in-plane orientation texture. In our calculations, the concentration of adsorbed particles plays a role similar to the flow: it is an agent that can perturb the uniform texture. However, there are differences: first, the shear introduces a preferred direction along the whole system, while the anchoring induced by the adsorbed particles is more local; in second place, the bright and dark bands observed in our system correspond to an in-plane variation of the orientation of the liquid crystal and no out-of-plane component of the director is observed.

Figure 4 shows the correlation length $L_{D}(t)$ as a function of time, for different concentrations of particles (Table I reports a small set of the data, including results at very long times for the concentration $\phi=0.33$ ). A small number of particles (see the data for $\phi=0.08$ and $\phi=0.17$ ) can slow down the relaxation process considerably. In these cases, the surfaces impose a late stage, quasiuniform texture that is slightly perturbed by the presence of a few topological defects attached to the particles. The growth of the correlation

TABLE I. Average domain size $L_{D}$ as a function of time, for the bulk LC, and five confined systems with increasingly higher particle concentration $\phi$. [Ellipsis indicate that, at the corresponding time, the system has relaxed to a monodomain state where $C(x, t)$ never decays below $1 / 2$.]

\begin{tabular}{rrrrrrr}
\hline \hline \multicolumn{1}{c}{$t\left(\tau_{0}\right)$} & Bulk & $\phi=0$ & $\phi=0.08$ & $\phi=0.17$ & $\phi=0.33$ & $\phi=0.41$ \\
\hline 1.4 & 4.0 & 4.0 & 4.0 & 4.0 & 4.0 & 4.0 \\
5.6 & 4.7 & 4.7 & 4.7 & 4.7 & 4.7 & 4.7 \\
17.5 & 8.4 & 7.7 & 7.7 & 7.7 & 7.7 & 7.7 \\
70 & 14.6 & 14.2 & 14.2 & 14.2 & 14.2 & 14.2 \\
105 & 17.9 & 17.5 & 17.2 & 17.2 & 17.2 & 17.2 \\
175 & 23.7 & 24.5 & 23.0 & 22.3 & 20.5 & 23.1 \\
280 & 29.6 & 57.7 & 35.8 & 30.7 & 30.3 & 31.0 \\
350 & 32.7 & $\cdots$ & 55.9 & 38.7 & 31.4 & 35.1 \\
525 & & & $\cdots$ & $\cdots$ & 41.3 & 42.4 \\
735 & & & & & 49.3 & \\
1540 & & & & & 63.9 & \\
2345 & & & & & 69.8 & \\
4900 & & & & & 80.2 & \\
6300 & & & & & 80.0 & \\
7700 & & & & & 80.0 & \\
10500 & & & & & &
\end{tabular}




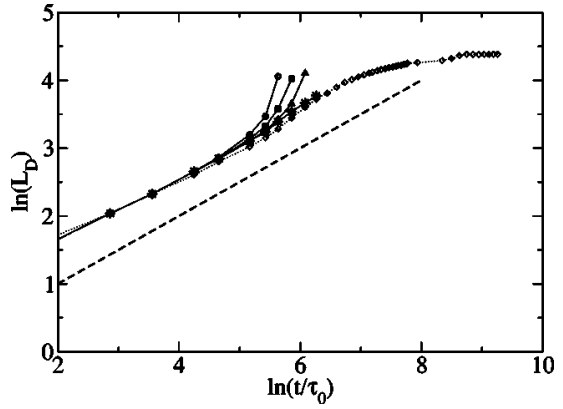

FIG. 5. Growth of domain size $L_{D}(t)$ on a logarithmic scale without particles (circles), with $\phi=0.08$ (squares), with $\phi=0.17$ (triangles), and with $\phi=0.33$ (star). The diamonds represent long-time runs for a configuration with $\phi=0.33$, averaged over 30 initial configurations of the liquid crystal. The dotted line represents the scaling law $L_{D}(t) \sim t^{1 / 2}$.

length $L_{D}(t)$ is similar to the case without bound particles, with a departure from the scaling law $L_{D}(t) \sim t^{1 / 2}$ setting in at long times, as shown in Figs. 4 and 5.

At a critical concentration of particles (see Fig. 4 for $\phi$ $=0.33$ ), perturbations created by the bound particles are such that the substrate properties are no longer able to induce a uniform nematic texture. The dynamics of the system is governed by $L_{D}(t) \sim t^{1 / 2}$ over a wide range of time, reflecting that no preferred orientation is promoted by the surfaces. We have investigated dynamics at very long times at this critical concentration. Results are reported in Fig. 5 on a logarithmic scale. Even at late stages, the correlation function $C(x, t)$ is never uniform. $C(x, t)$ is frozen and exhibits a shape similar to that observed in Fig. 2(b) for $t=630 \tau_{0}$. The correlation length $L_{D}(t)$ grows until it saturates at a finite value $(80 \xi)$, as indicated in Fig. 5. At this concentration of particles, the final structure corresponds to a disordered state devoid of uniform orientational order: As one traverses the cell along the $x$ direction, the director oscillates, giving rise to alternating white and black bridges between the two substrates (see Fig. 3).

Experimentally, the optical signatures for low protein coverages ${ }^{1-4}$ indicate a uniform texture slightly perturbed by topological defects attached to the proteins. At higher protein densities, the optical signatures are those of a nematic texture characterized by a multidomain structure. These observations are consistent with the results of our simulations, which reveal a multidomain structure characterized by slow dynamics. For a high particle concentration $(\phi=0.66)$, we observe final states having again uniform orientational order in the cell, but with a director that is perpendicular to the substrates. The occurrence of these equilibrium structures results from the assumption of homeotropic anchoring at the surface of the particles. Similar equilibrium structures have been observed for viruses captured by surfaces. ${ }^{4}$ This comparison between simulations and experiments suggests that, in these experiments, the liquid crystal may be oriented perpendicularly to the surface of the viruses; at high densities, the viruses impose a global orientation perpendicular to the substrates.

\section{CONCLUSION}

The results presented in this paper indicate that as long as the surface properties are not transmitted towards the middle of the cell, the dynamical correlation function is characterized by an average domain size $L_{D}$ that grows as $L_{D}(t) \sim t^{\phi}$, with $\phi \approx 0.50 \pm 0.02$. This $t^{1 / 2}$ behavior is in agreement with experimental observations ${ }^{8,9}$ and with recent numerical investigations that include back-flow effects. ${ }^{15}$

In the absence of adsorbed particles, the surfaces induce a long-range orientational order throughout the entire cell. For a small number of particles adsorbed at the solid surfaces, a similar behavior is observed but the relaxation towards a long-range uniform orientation order is delayed. As the concentration of particles increases, these effects become increasingly pronounced until, at a critical concentration, no monodomain orientational order is observed. At this concentration $(\phi=0.33)$, the dynamics obey a scaling law of the form $L_{D}(t) \sim t^{1 / 2}$ until the growth of domains is retarded at late stages. At these late stages, the system becomes trapped in a metastable state where configurations are characterized by a disordered texture with finite domain sizes.

Several questions are now open for further investigations: first, for a given geometry $L_{x} \times L_{y}$ of the nematic film, what determines the critical concentration $\phi$ that prevents the establishment of a uniform texture dictated by the anchoring of the confining substrates? Second, would it be possible to enhance the sensitivity of LC biosensors by choosing specific configurations of the binding sites that enhance the contrast between the presence and absence of the adsorbed particles? The coarse-grained theory used in this work seems to be well suited to answer these questions.

\section{ACKNOWLEDGMENTS}

This work was supported by the National Science Foundation through the University of Wisconsin's MRSEC on Nanostructured Interfaces.

${ }^{1}$ V. K. Gupta, J. J. Skaife, T. B. Dubrovsky, and N. L. Abbott, Science 279, 2077 (1998)

${ }^{2}$ J. A. van Nelson, S. R. Kim, and N. L. Abbott, Langmuir 18, 5031 (2002).

${ }^{3}$ R. R. Shah and N. L. Abbott, Langmuir 19, 275 (2003).

${ }^{4}$ Y.-Y. Luk, M. L. Tingey, D. J. Hall, B. A. Israel, C. J. Murphy, P. J. Bertics, and N. L. Abbott, Langmuir 19, 1671 (2003).

${ }^{5}$ S. Grollau, E. B. Kim, O. Guzmán, N. L. Abbott, and J. J. de Pablo, J. Chem. Phys. 119, 2444 (2003).

${ }^{6}$ O. Guzmán, E. B. Kim, S. Grollau, N. L. Abbott, and J. J. de Pablo, Phys. Rev. Lett. 91, 235507 (2003).

${ }^{7}$ E. B. Kim, O. Guzmán, S. Grollau, N. L. Abbott, and J. J. de Pablo, J. Chem. Phys. 121, 1949 (2004).

${ }^{8}$ A. P. Y. Wong, P. Wiltzius, and B. Yurke, Phys. Rev. Lett. 68, 3583 (1992).

${ }^{9}$ A. P. Y. Wong, P. Wiltzius, R. G. Larson, and B. Yurke, Phys. Rev. E 47, 2683 (1993)

${ }^{10}$ R. E. Blundell and A. J. Bray, Phys. Rev. A 46, R6154 (1992).

${ }^{11}$ M. Zapotocky, P. M. Goldbart, and N. Goldenfeld, Phys. Rev. E 51, 1216 (1995).

${ }^{12}$ A. Bhattacharya, M. Rao, and A. Chakrabarti, Phys. Rev. E 53, 4899 (1996).

${ }^{13}$ N. V. Priezjev and R. A. Pelcovits, Phys. Rev. E 66, 051705 (2002)

${ }^{14}$ J. I. Fukuda, Eur. Phys. J. B 1, 173 (1998).

${ }^{15}$ C. Denniston, E. Orlandini, and J. M. Yeomans, Phys. Rev. E 64, 21701 (2001).

${ }^{16}$ X.-1. Wu, W. I. Goldburg, M. X. Liu, and J. Z. Xue, Phys. Rev. Lett. 69, 470 (1992). 
${ }^{17}$ P. G. de Gennes and J. Prost, The Physics of Liquid Crystals (Clarendon, Oxford, 1993)

${ }^{18}$ A. N. Beris and B. J. Edwards, Thermodynamics of Flowing Systems: With Internal Microstructure (Oxford University Press, New York, 1994).

${ }^{19}$ J. Fukuda and H. Yokoyama, Eur. Phys. J. E 4, 389 (2001).

${ }^{20}$ S. Grollau, N. L. Abbott, and J. J. de Pablo, Phys. Rev. E 67, 051703 (2003).

${ }^{21}$ P. Galatola, J.-B. Fournier, and H. Stark, Phys. Rev. E 67, 031404 (2003).

${ }^{22}$ V. K. Gupta and N. L. Abbott, Science 276, 1533 (1997).

${ }^{23}$ M. Doi, J. Polym. Sci., Polym. Phys. Ed. 19, 229 (1981).

${ }^{24}$ A. D. Rey and T. Tsuji, Macromol. Theory Simul. 7, 623 (1998).

${ }^{25}$ B. J. Edwards and A. N. Beris, J. Rheol. 33, 1189 (1989).

${ }^{26}$ M. J. Stephen and J. P. Straley, Rev. Mod. Phys. 46, 617 (1974).

${ }^{27}$ J. Nehring and A. Saupe, J. Chem. Phys. 56, 5527 (1972).
${ }^{28}$ N. Schopohl and T. J. Sluckin, Phys. Rev. Lett. 59, 2582 (1987).

${ }^{29}$ Simulations are performed with $D=0.35, L_{1}=0.55$, and $A=1$, representative of low molecular weight liquid crystals such as pentylcyanobiphenyl $(5 \mathrm{CB})$. The equilibrium nematic scalar order parameter after the quench is equal to $S_{\text {bulk }}=0.809$.

${ }^{30}$ G. Barbero and G. Durand, J. Appl. Phys. 69, 6968 (1991).

${ }^{31}$ K. Hiltrop and H. Stegemeyer, Liq. Cryst. Ordered Fluids 4, 515 (1984).

${ }^{32}$ J. Fang, U. Gehlert, R. Shashidhar, and C. M. Knobler, Langmuir 15, 297 (1999).

${ }^{33}$ L. A. Tecero Espinoza, K. R. Schumann, Y.-Y. Luk, B. A. Israel, and N. L. Abbott, Langmuir 20, 2375 (2004).

${ }^{34}$ J. M. Brake, M. K. Daschner, Y.-Y. Luk, and N. L. Abbott, Science 302, 2094 (2003) 